\title{
LUNG CANCER AND PULMONARY TUBERCULOSIS - A COMPARATIVE POPULATION-GENETIC STUDY
}

\author{
Pešut $\mathrm{DP}^{1,2, *}$, Marinkovic $\mathrm{DM}^{3}$
}

\begin{abstract}
*Corresponding Author: Dragica P. Pešut, Institute of Lung Diseases and Tuberculosis, Research and Epidemiology Department, Visegraska 26/20, 11000 Belgrade, Serbia; Tel.: +381-11-3615561; Fax: +381-11-268-1591; E-mail: dragica.pesut@gmail.com
\end{abstract}

\section{ABSTRACT}

Several host genes proven to contribute to active tuberculosis (TB) and some of the localized major susceptibility loci, which influence lung cancer (LC) risk, are of considerable scientific interest, but do not confer high enough risk to be clinically relevant. Assuming that these diseases are genetically controlled, we hypothesized that retreat from optimal homozygosity level, as well as a changed variability among the patients, could be the populationgenetic parameter for prediction of illness. We performed a homozygous-recessive-characters (HRCs) test based analysis of the presence, distribution and individual combination of 23 selected geneticallycontrolled morpho-physiological traits in groups of LC patients, patients with pulmonary TB and healthy control subjects. This study showed: $\boldsymbol{i}$ ) a statistically significant difference of the middle values of genetic homozygosity between both patients groups and the control group, ii) differences in the type of distribution, and iii) differences in the presence of certain individual combinations of such traits. The frequency of blood group $\mathrm{O}$ was significantly decreased in the TB group compared to the general population.

1 School of Medicine University of Belgrade, Belgrade, Serbia

2 Clinical Centre of Serbia, Institute of Lung Diseases and Tuberculosis, Research and Epidemiology Department, Belgrade, Serbia

3 Serbian Academy of Sciences and Arts, Belgrade, Serbia
According to their population-genetic structure, LC patients, TB patients and healthy controls represent three different groups. The retreat from optimal homozygosity level towards decrease that we found in both LC and TB patients support the influence of a dominant factor in development of these diseases.

Key words: Genetic predisposition; Lung cancer (LC); Tuberculosis (TB); Phenotype; Marker; Population genetic

\section{INTRODUCTION}

Lung cancer (LC) is the most frequent type of cancer in men [1]. Despite therapeutic possibilities, this devastating disease remains the leading cause of cancer death in the world with more than 1.2 million new cases diagnosed every year [2]. Its etiology has been mentioned as an example of ecogenetics in oncology with tobacco smoking as the major risk factor [3]. Lung cancer is an etiologically complex disease in which multiple genes are involved in the pathogenesis via different pathways $[4,5]$. Some of the major susceptibility loci which influence LC risk have been localized [5-10]. Such susceptibility genes are of considerable scientific interest, but do not confer high enough risks to be clinically relevant [6].

Tuberculosis (TB), an infectious disease caused by Mycobacterium tuberculosis complex, was declared a global emergency in 1993 and is still a problem due to the increasing number of immunedeficient persons in the world and the development of multi-drug resistant TB $[11,12]$. It is estimated 
that one-third of the world population is infected with TB bacillus $[11,12]$. Often the antibodies present in the human organism are strong enough to prevent the establishment of the disease. Although a variety of studies have suggested that host genetic factors may be a key to determining susceptibility/ resistance to $M$. tuberculosis, those that have assessed variants of the natural resistance-associated macrophage protein 1 gene (NRAMP1) and their association with TB in humans have yielded conflicting results [13-19]. It is likely that NRAMP1 polymorphisms may be associated with progression to severe forms of pulmonary TB rather than with susceptibility to M. tuberculosis infection $[18,19]$. Recent findings suggest that persons carrying the monocyte chemo-attractant protein-1 (MCP-1) genotype GG produce high concentrations of MCP-1, which inhibits production of IL-12p40 in response to $M$. tuberculosis and increases the likelihood that M. tuberculosis infection will progress to active pulmonary TB [20]. Thus, despite the known infectious cause, the etiology of TB remains complex, and several host genes have been shown to contribute to the development of clinical disease $[19,21,22]$. Our previous population-genetic research indicated a possible role for a dominant factor influence in TB [17]. Although the molecular basis of genetic predisposition to pulmonary TB in adults remains largely elusive, a genome-wide linkage study provided direct molecular evidence that the genetic component involves at least one major locus with a dominant susceptibility allele [21]. Genes which are involved in susceptibility to TB include: $12 \mathrm{q} 14$, 2q35, 15q11-q13 (OMIM numbers 607948, 607949, 300259), and 8q12-q13 [23].

In population-genetic investigations, a great number of morpho-physiological characters serve as genetic markers. Individual variation is a phenomenon, which can easily be noticed when we carefully observe the same species of organisms. Thus, human populations vary in regard to face shape, skin pigmentation, height, hair color, blood types as well as functions and abilities. Some of these characters are expressed in a recessive or dominant type and are called qualitative traits [24]. Thus, blue eyes are a recessive trait, brown eyes are a dominant one, and inability to recognize the bitter taste of phenylthio-carbamide (PTC), left-handedness and fixed ear lobe are recessive traits. We applied this method to estimate the proportion of a variety of homozygousrecessive characters (HRCs) tests in two groups of patients with respiratory diseases and in a healthy control group.

We hypothesized that there is a difference in the population-genetic structure of LC patients, patients who develop active pulmonary TB and healthy controls. Our aims were $\boldsymbol{a}$ ) to analyze and compare the population-genetic structure of these three groups using a number of qualitative morphophysiological traits; $\boldsymbol{b}$ ) to evaluate the degree of genetic homozygosity in these three groups according to the frequency of homozygous-recessive traits; $\boldsymbol{c}$ ) to investigate the frequency of independent genetic determinants such as the ABO blood type in these three groups.

\section{MATERIAL AND METHODS}

Study Group. We studied 100 LC patients, 50 pulmonary TB patients and 50 healthy non sibling controls matched in sex, age and smoking habits. All diagnoses were established at the Clinical Centre of Serbia Institute of Lung Diseases and Tuberculosis in Belgrade, Serbia. Diagnosis of LC was based on histological examination of the tissue obtained during bronchoscopy, by fine needle aspiration biopsy or open lung biopsy. Pulmonary TB was confirmed by positive results of sputum culture on Löwenstein-Jenssen medium and/or by histology. Control group consisted of spouses/partners of patients or employees of the Institute of Lung Diseases and Tuberculosis in Belgrade, Serbia, with 20 or more years exposure at the working place, who had not developed TB or LC by the time of the study.

Methods. Establishing genetic homozygosity in humans is a very delicate assignment, because we know only a small number of loci with allelogenes that determine an exact biochemical process [25]. Knowing the type of inheritance and variability we can see that series of morpho-physiological traits are under control of one small number of genes. Some homozygously-recessive traits of the head and neck region are: straight, soft hair and blond hair (OMIM numbers 139450 and 210750), inability to roll, fold and curve the tongue (OMIM number 189300), insensitivity to PTC (gene location 7q35-q36, OMIM number 607751), color blindness (gene location Xq28, OMIM number 303800), while the others are 
clearly expressed in human arms and legs like hand clasping pattern, left handedness (gene location 2p12-q22, OMIM number 139900), distal or proximal hyper-extensibility of the thumb, index finger shorter than the ring finger (OMIM number 136100), and so on [23]. Some of the traits are still a matter of intensive research such as blue eyes, previously found gene location 19p13.1-q13.11 (OMIM number 227240) and newly discovered at the $15 \mathrm{q} 13.1$ as the predominant region involved in human iris color
[26]. Using this information, several authors of Belgrade population-genetic studies have studied the distribution and frequency of a series of extremely expressed recessive traits to estimate individual and group differences, i.e., comparison between ill and healthy individuals, pupils with special needs, carriers of different blood types, members of different ethnic groups, etc. [25,27-31].

We examined a total of 23 specific HRCs (listed in Table 1), characterized by Marinkovic et al.

Table 1. Comparison of frequencies of homozygously recessive traits between two groups of patients: affected by LC, affected by TB, and control (healthy persons) (23 recessive alleles).

\begin{tabular}{|c|c|c|c|c|c|c|}
\hline $\begin{array}{l}\text { LC Affected }-\chi \mathrm{hrc} / 23=5.86 \pm 0.25 \\
\text { TB Affected }-\chi \mathrm{hrc} / 23=6.12 \pm 0.28 \\
\text { Controls }-\chi \mathrm{hrc} / 23=7.06 \pm 0.26\end{array}$ & $\begin{array}{c}\text { LC }(n=100): \\
\text { Controls }(n=50)\end{array}$ & $\begin{array}{l}\mathrm{LC}(n=100): \\
\mathrm{TB}(n=50)\end{array}$ & $\begin{array}{c}\text { TB }(n=50): \\
\text { Controls }(n=50)\end{array}$ & $\begin{array}{c}\text { LC: } \\
\text { Controls }\end{array}$ & $\begin{array}{l}\text { LC: } \\
\text { TB }\end{array}$ & $\begin{array}{c}\text { TB: } \\
\text { Controls }\end{array}$ \\
\hline Homozygous Recessive Trait & $\chi^{2}$ & $\chi^{2}$ & $\chi^{2}$ & Fst & Fst & Fst \\
\hline Blond hair & 1.50 & 2.66 & 0.11 & 0.0026 & 0.0050 & 0.0004 \\
\hline Soft hair & $4.00^{\mathrm{a}}$ & 1.00 & 0.60 & $0.0302^{\mathrm{a}}$ & 0.0123 & 0.0040 \\
\hline Straight hair & 1.30 & 0.03 & 0.89 & 0.0163 & 0.0019 & 0.0050 \\
\hline Even scalp & 0.94 & 2.11 & 0.19 & 0.0037 & 0.0086 & 0.0017 \\
\hline Light skin & $9.30^{\mathrm{b}}$ & 0.69 & $4.00^{\mathrm{a}}$ & $0.0635^{\mathrm{c}}$ & 0.0144 & 0.0153 \\
\hline Attached earlobe & $12.25^{\mathrm{c}}$ & 0.33 & $21.30^{\mathrm{c}}$ & $0.0531^{\mathrm{b}}$ & 0.0000 & $0.0530^{\mathrm{b}}$ \\
\hline Ear without Darwin's lump & 0.25 & 0.25 & 0.00 & 0.0016 & 0.0016 & 0.0000 \\
\hline Narrow nostrils & $40.50^{\mathrm{c}}$ & $32.00^{\mathrm{c}}$ & 0.10 & $0.0531^{\mathrm{b}}$ & 0.0445 & 0.0004 \\
\hline Blue eyes & 0.33 & 0.07 & 0.75 & 0.0100 & $0.0016^{\mathrm{b}}$ & 0.0036 \\
\hline Hyperextensibility of the arm joint & 0.05 & 1.14 & 0.64 & 0.026 & 0.0123 & 0.0036 \\
\hline Pectus carinatum & 1.00 & 1.00 & 1.00 & $0.0752^{\mathrm{c}}$ & 0.1111 & 0.0060 \\
\hline Notability of tongue rolling & 0.00 & 1.00 & 2.00 & $0.1363^{\mathrm{c}}$ & $0.0023^{\mathrm{c}}$ & $0.1100^{\mathrm{c}}$ \\
\hline $\begin{array}{l}\text { Notability of transverse tongue } \\
\text { rolling }\end{array}$ & $4.26^{\mathrm{a}}$ & 0.04 & 3.26 & 0.0176 & 0.0001 & 0.0150 \\
\hline $\begin{array}{l}\text { Notability of recognizing the bitter } \\
\text { PTC taste }\end{array}$ & 3.76 & 3.76 & 0.00 & 0.0122 & 0.0122 & 0.0000 \\
\hline Color blindness & 2.00 & 2.00 & 0.00 & $0.0752^{\mathrm{c}}$ & 0.0752 & 0.0000 \\
\hline Left handedness & $4.50^{\mathrm{a}}$ & $4.50^{\mathrm{a}}$ & 0.00 & 0.0087 & $0.0087^{\mathrm{c}}$ & 0.0000 \\
\hline Speech disabilities & 0.20 & $5.00^{\mathrm{a}}$ & $16.00^{\mathrm{c}}$ & 0.0040 & 0.0696 & $0.0370^{\mathrm{c}}$ \\
\hline Thumb distal hyperextensibility & 1.28 & 0.16 & 2.66 & 0.0180 & $0.0010^{c}$ & 0.0004 \\
\hline $\begin{array}{l}\text { Proximal thumb joint } \\
\text { hyperextensibility }\end{array}$ & $8.00^{\mathrm{b}}$ & 1.00 & $25.00^{\mathrm{c}}$ & $0.2048^{c}$ & 0.0752 & $0.1090^{c}$ \\
\hline Index finger longer than 4 th finger $^{\mathrm{d}}$ & $33.90^{\mathrm{c}}$ & 1.23 & $17.00^{\mathrm{c}}$ & $0.1406^{\mathrm{c}}$ & $0.0144^{c}$ & $0.0680^{\mathrm{c}}$ \\
\hline $\begin{array}{l}\text { Hairlessness of the hand middle } \\
\text { finger phalange }\end{array}$ & 0.20 & 0.72 & 1.80 & 0.0026 & 0.0215 & 0.0110 \\
\hline Right thumb over left thumb & 1.96 & 3.52 & 0.17 & 0.0083 & $0.0151^{\mathrm{a}}$ & 0.0004 \\
\hline Three strings in the wrist & 0.11 & 0.44 & 0.10 & 0.0018 & 0.0053 & 0.0009 \\
\hline$\Sigma \chi^{2}$ & $131.59^{c}$ & $64.69^{c}$ & $97.57^{\mathrm{c}}$ & - & - & - \\
\hline
\end{tabular}

${ }^{\mathrm{a}} p<0.05 ;{ }^{\mathrm{b}} p<0.01 ;{ }^{\mathrm{c}} p<0.001 ;{ }^{\mathrm{d}}$ in males, opposite in females. 
[27,28], in each subject from the three groups. The study was approved by the Ethics Committee of the Clinical Centre of Serbia, Belgrade, Serbia, and its purpose was described to all the individuals in the three groups. Ability to recognize the bitter taste of PTC was tested following original instructions of Harris and Kalmus [32] and the color-blindness test was performed according to Ishihara [33].

Statistics. We used a combination of $\chi 2$ test, Turkey's test, non parametric variance analysis, two-by-two $\chi^{2}$ tabs, equal proportion test, and Wahlund's variance - the inter-population fixation index $\left(\mathrm{F}_{\mathrm{st}}\right)$, in a stepwise manner. Recessive allele frequencies (q) were determined according to the formula: $\mathrm{q}=\sqrt{\mathrm{R}} / \mathrm{N}(\mathrm{R}=$ number of the individuals with recessive character; $\mathrm{N}=$ number of individuals in population). Wahlund's variance $\left(\mathrm{F}_{\mathrm{st}}\right)$ was determined according to the formula: $\mathrm{F}_{\mathrm{st}}=$ (qi $\left.q^{\prime}\right)^{2} / q^{\prime}\left(1-q^{\prime}\right)(q i=$ the frequency of the i-allele in the group; $q^{\prime}=$ average frequency of the allele).

\section{RESULTS}

We found significant difference in average number of the HRCs between controls and each of the patients groups, with decreased number in the patients $(\mathrm{LC}=5.86 \pm 0.25$; $\mathrm{TB}=6.12 \pm 0.28$; control $=7.06 \pm 0.26$ ), the total cumulative $\chi^{2}$ dif- ference $(p<0.001)$ and frequency distribution $(p$ $=0.0027)$. The list and degree of the significance found for each of the studied HRCs in patients and controls are shown in the Table 1. The table also illustrates the values of the interpopulation fixation index (Fst) in the frequency trend of the recessive phenotypes in the analyzed groups. The particular analysis suggests that the both patients' groups represent different samples when compared to healthy controls. The difference is more expressed in LC patients, whose retreat toward lower degrees of homozygosity is more evident. The two patient groups also differ between themselves, but the difference is not significant in terms of the majority of the parameters investigated. Turkey's test and non parametric variance analysis confirmed this $\left(\mathrm{t}_{\mathrm{C}: \mathrm{TB}}=2.47, p\right.$ $\left.<0.05 ; \mathrm{t}_{\mathrm{C}: \mathrm{LC}}=3.35, p<0.01 ; \mathrm{t}_{\mathrm{TB}: \mathrm{LC}}=0.63, p>0.05\right)$. The HRCs frequencies in the three analyzed groups are shown in Figure 1. Homozygous-recessive characters frequency variation ranged from three to 10 in TB patients, from two to 11 in LC patients, and from three to 12 in controls. The frequency distribution is shown in Figure 2.

The frequencies of $\mathrm{ABO}$ blood types in the group of LC patients were similar to the average proportion in the Serbian population, while the frequency of blood type $\mathrm{O}$ was significantly decreased in the TB patients group $(p<0.05)$. In this latter group, the

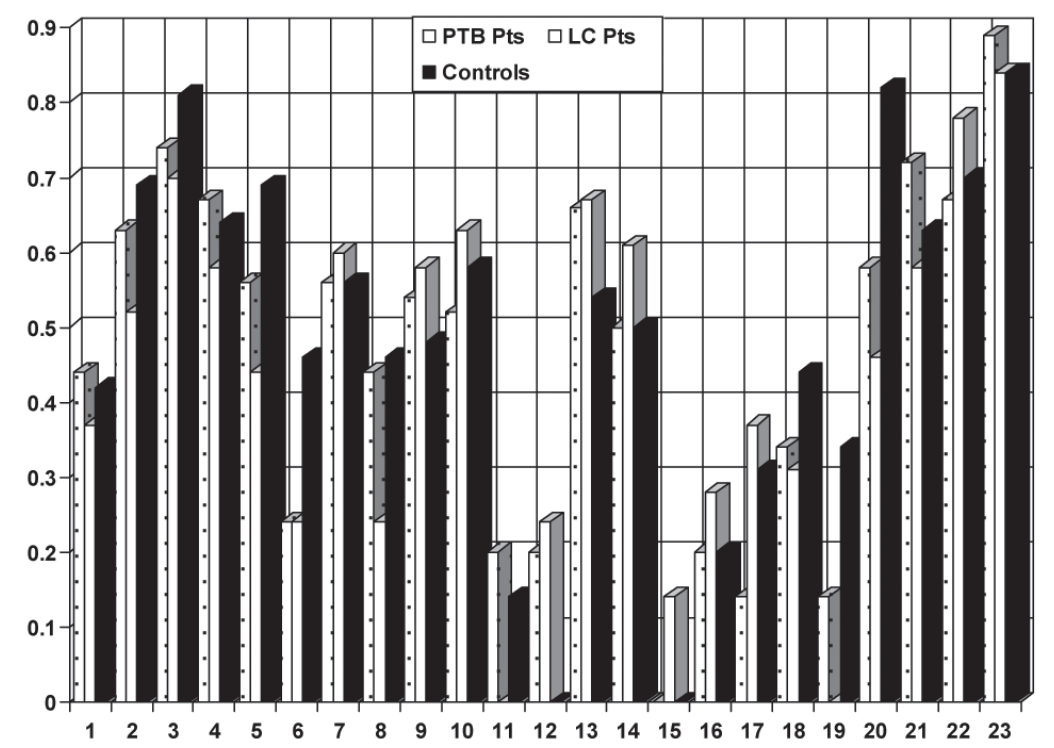

Figure 1. Recessive allele frequency in the pulmonary tuberculosis (PTB) patients, lung cancer (LC) patients and healthy controls. The recessive alleles (1-23) are listed in Table 1. 


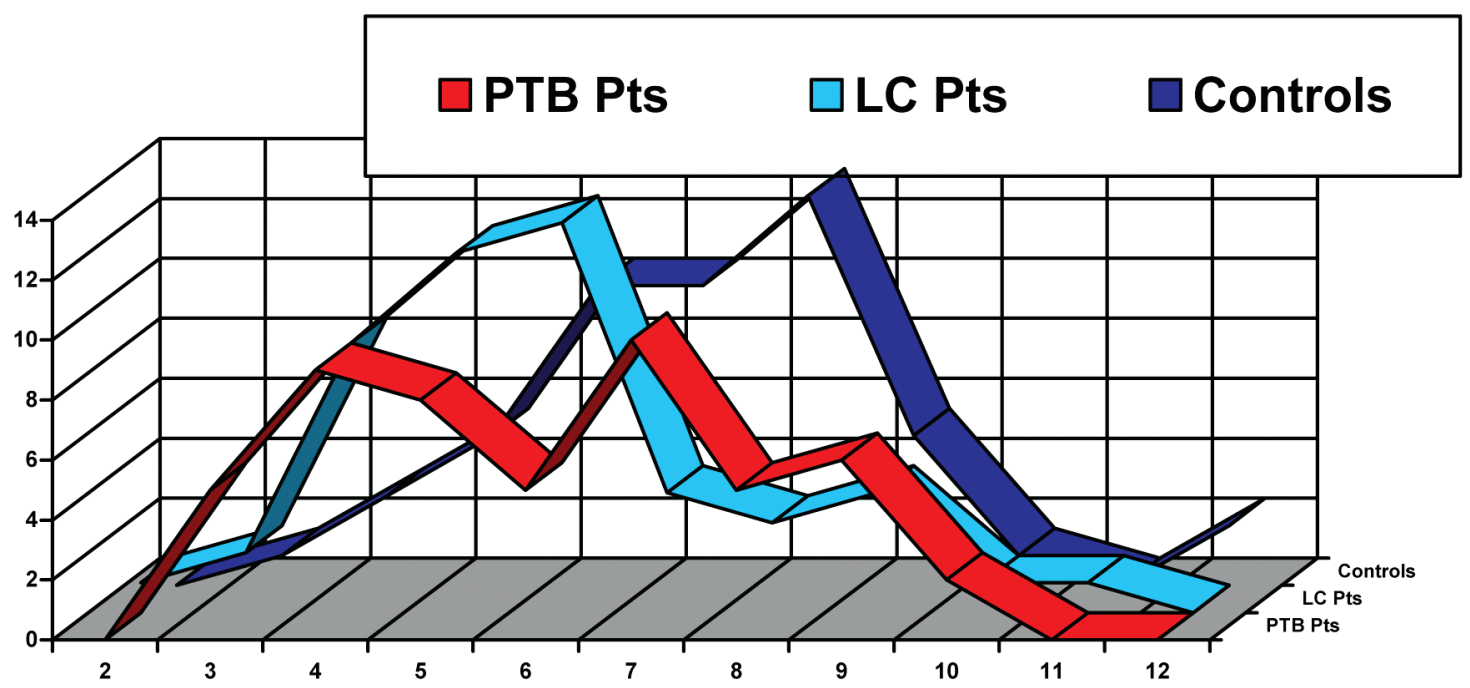

Figure 2. Frequency distribution of 23 HRCs in pulmonary TB (PTB) patients, lung cancer (LC) patients and controls. Controls: PTB $p<0.05, p=0.0027$; Controls: LC $p<0.01$; PTB: LC $p>0.05$.

proportion of blood type B was increased, but not significantly (Figure 3).

\section{DISCUSSION}

This study showed a statistically significant difference of the middle values of genetic homozygosity, differences in the type of distribution and of the presence of certain individual combinations of such traits. Significantly decreased HRC number in both patients' groups could be a manifestation of their decreased degree of homozygosity and might support dominant factor influence in predisposition to TB and to LC. While the molecular genetic research on Moroccan multiplex families supports this for TB [21], a new research on MDM2 promoter polymorphisms as associated risk for LC showed contradiction to previous reports [8]. The results of our study, together

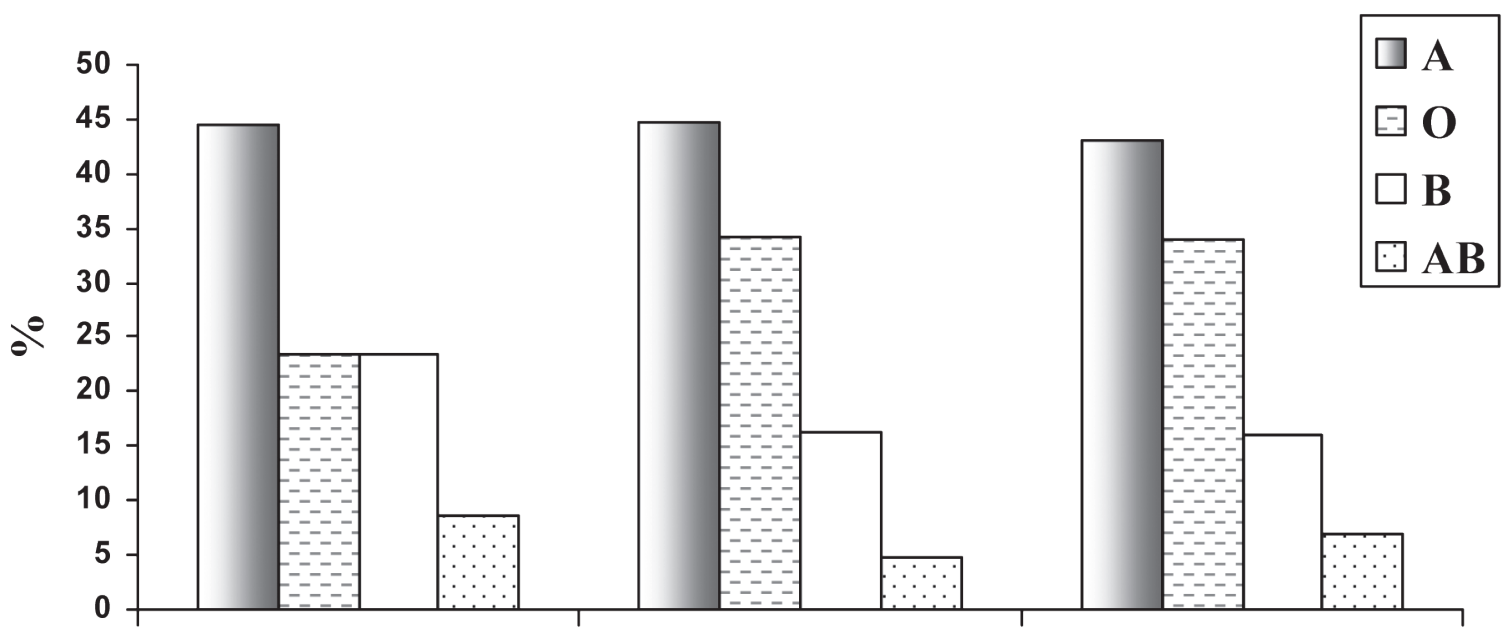

PTB Pts

LC Pts

General population

Figure 3. The frequency of blood type $\mathrm{O}$ was significantly decreased in the TB patients group compared to the general population of Serbia $(p<0.05)$. 
with previous research based on the same methodology, show that any retreat from optimal homozygosity degree, whether decrease or increase, points to the possibility of changed genetic-physiological organism homeostasis and its altered reaction norm that leads to illness [17,25,27-31]. Namely, apart from LC and TB, decreased homozygousity was found in asthma, acute appendicitis, while increased homozygosity level has been shown in diabetes mellitus, congenital hip dislocation and Balkan endemic nephropathy, to mention just a few [17,25,27-31]. Recent direct molecular evidence that TB involves a genetic component of at least one major locus with a dominant susceptibility allele, also support our findings [21]. While in the mentioned study, a decrease of logarithm of the odds (LOD) score for TB was found in the 19p12 gene location, previously connected to blue eye color, a new research of a Dutch group indicated that $15 \mathrm{q} 13.1$ was the predominant region involved in human iris color [26]. This latter could bring a new light to our earlier finding that eye color and hair color in TB patients have a characteristic expression and independent inheritance, which might make closer the particular genome region responsible for TB susceptibility [17]. The new eye color location also correlates with the finding of a locus at 15q11-q13 (OMIM number 300259) for susceptibility to TB. Only interdisciplinary approach could make it possible to put into connection eye color-related findings aimed to improve forensic analysis to the important field of susceptibility to TB. Similarly, while epidemiologic and clinical studies connected TB with diabetes mellitus long ago, susceptibility regions at $2 q 34-2 q 36.2$ have been associated with TB and diabetes mellitus [23].

We found significantly decreased O blood type in TB patients. Numerous studies have shown the frequency of ABO blood types differ in groups of patients or subjects $[13,17,28]$. We assume that certain connection between a predisposition to TB and some of the ABO blood types exists, and this is in correlation with the previous findings $[14,17]$. Our study on associated diseases showed significantly decreased number of palatal tonsillectomies in both LC and TB patients, increased the average age at tonsillectomy in LC patients compared to controls, and decreased frequency of allergic diseases in the LC group [34]. Both the mentioned results on independent genetic markers in LC and TB need further investigation with a larger sample size and multi center multi disciplinary research.

There were possible limitations in our study such as the list and number of chosen HRCs and their correct identification in the examined persons. The list of traits was developed as the result of the experiences of the authors of the HRCs test in its previous application in several other studies [27-31]. Since 23 homo-recessive traits were analyzed in each individual, both in patients groups and controls, this multiplied the total number of tested traits to 4,600 in total, and influenced the statistical power of the test. Thus, even a smaller number of patients and controls would be sufficient in this kind of investigation. When it comes to possible errors generated by subjectively different identification of the traits, it has been taken to a minimum having only one person to detect them in all the examinees.

Although enormous efforts to find out a single dominant factor both in LC and TB have been done, especially during the last decade, it is more probable that not a single gene but a pleiotropic effect of few (many) plays a role in development of these diseases. The results of our study, according to i) HRCs average number, ii) gene allele frequencies, iii) total cumulative $\chi 2$ difference, and $i v)$ frequency distribution, showed that both patients' groups present different samples when compared to healthy controls, according to their population-genetic structure. That means that persons in these groups may give a different response to similar environmental agents when compared to healthy ones. The population-genetic approach in this comparative analysis may serve as paradigm of successful cooperation between clinicians, epidemiologists and laboratory scientists in the pursuit of the understanding of cancer etiology and predisposition, and susceptibility to TB in humans. The described methodology can be used in further improved analyses, with hope that it can be applied in as an early prognosis for susceptibility to a variety of diseases.

\section{REFERENCES}

1. Skuladottir H, Olsen JH. Epidemiology of lung cancer. Eur Respir Mon 2001; 6(17): 1-12.

2. Wynder LE. Epidemiology of smoking and lung cancer: a historical perspective. Lung Cancer 1994; 11(Suppl 2): 1. 
3. Mulvihill JJ. Host factors in human lung tumors: an example of ecogenetics in oncology. J Natl Cancer Inst 1976; 57(1): 3-7.

4. Kaye FJ, Kim JW. Inactivation of the $R b$ gene in human cancer. Lung Cancer 1994; 11(Suppl 2): 8-9.

5. Bailey-Wilson JE, Amos CI, Pinney SM, Petersen GM, de Andrade M, Wiest JS, Fain P, Schwartz AG, You M, Franklin W, Klein C, Gazdar A, Rothschild H, Mandal D, Coons T, Slusser J, Lee J, Gaba C, Kupert E, Perez A, Zhou X, Zeng D, Liu Q, Zhang Q, Seminara D, Minna J, Anderson MW. A major lung cancer susceptibility locus maps to chromosome 6q23-25. Am J Hum Genet 2004; 75(3): 460-474.

6. Vineis P. Individual susceptibility to carcinogens. Oncogene 2004; 23(38): 6477-6483.

7. Li G, Wang LE, Chamberlain RM, Amos CI, Spitz MR, Wei Q. p73 G4C14-to-A4T14 Polymorphism and risk of lung cancer. Cancer Res 2004; 64(19): 6863-6866.

8. Li G, Zhai X, Zhang Z, Chamberlain RM, Spitz MR, Wei Q. MDM2 gene promoter polymorphisms and risk of lung cancer: a case-control analysis, Carcinogenesis 2006; 27(10):2028-2033.

9. Stankovic MM, Nestorovic AR, Tomovic AM, Petrovic-Stanojevic ND, Andjelic-Jelic MS, Dopudja-Pantic VB, Nagorni-Obradovic LjM, Mitic-Milikic MM, Radojkovic DP. TNF- $\alpha-308$ promotor polymorphism in patients with chronic obstructive pulmonary disease and lung cancer. Neoplasma 2009;56(4):348-352.

10. Kang JU, Koo SH, Kwon KC, Park JW, Kim JM. Identification of novel candidate target genes, including EPHB3, MASP1 and SST at 3q26.2-q29 in squamous cell carcinoma of the lung. BMC Cancer 2009; 9:237 (available at: http://www.biomedcentral.com/1471-2407/9/237).

11. Global tuberculosis control. Surveillance. Planning. Financing, WHO Report 2007, Geneva: World Health Organization, 2007.

12. Rieder HL, Ed. Interventions for Tuberculosis Control and Elimination. Paris: International Union against Tuberculosis and Lung Disease. 2002; 1-169.

13. Volkova KI, Blinetskaia ZS, Fateev IN. Genetic blood markers of the $\mathrm{ABO}$ system in patients with pulmonary tuberculosis in relation to ethnic origin. Probl-Tuberk 1991; 10: 55-58.
14. Kaminskaja GO, Zhukova NL, Naumov VN. Interrelations between genetically determined haptoglobin types and course of the postoperative period of chronic forms of pulmonary tuberculosis. Probl-Tuberk 1989; 8: 3-7.

15. Remus N, Reichenbach J, Picard C, Rietschel C, Wood P, Lammas D, Kumararatne S, Casanova J-L. Impaired interferon $\gamma$-mediated immunity and susceptibility to mycobacterial infection in childhood. Pediatr Res 2001; 50(1): 8-13.

16. Casoni G, Caramori G, Papadopoulos NG, Johnston SL, Papi A. Gender and respiratory infections. Eur Respir Mon 2003; 8(25): 228-241.

17. Pesut D. Susceptibility to lung tuberculosis - a population-genetic study. Med Pregl 2004; 57(Suppl 1): 21-24.

18. Zhang W, Shao L, Weng X, Hu Z, Jin A, Chen S, Pang M, Chen ZW. Variants of the natural resistance-associated macrophage protein 1 gene (NRAMP1) are associated with severe forms of pulmonary tuberculosis. Clin Infect Dis 2005; 40(9): 1232-1236.

19. Bellamy R. Genetic susceptibility to tuberculosis. Clin Chest Med 2005; 26(2):233-246.

20. Flores-Villanueva PO, Ruiz-Morales JA, Song CH, Flores LM, Jo EK, Montano M, Barnes PF, Selman M, Granados J. A functional promoter polymorphism in monocyte chemoattractant protein-1 is associated with increased susceptibility to pulmonary tuberculosis. J Exp Med 2005; 202(12):1649-1658.

21. Baghdadi JE, Orlova M, Alter A, Ranque B, Chentoufi M, Lazrak F, Archane MI, Casanova JL, Benslimane A, Schurr E, Abel L. An autosomal dominant major gene confers predisposition to pulmonary tuberculosis in adults. J Exp Med 2006; 203(7): 1679-1684.

22. Davila S, Hibberd ML, Hari Dass R, Wong HE, Sahiratmadja E, Bonnard C, Alisjahbana B, Szeszko JS, Balabanova Y, Drobniewski F, van Crevel R, van de Vosse E, Nejentsev S, Ottenhoff $\mathrm{TH}$, Seielstad M. Genetic association and expression studies indicate a role of toll-like receptor 8 in pulmonary tuberculosis. PLoS Genet 2008; 4(10): e1000218.

23. The Online Mendelian Inheritance in Man database. Available at http://www.ncbi.nlm.nih.gov/ OMIM (last accessed April 24, 2009). 
24. Ayala FJ. Population and Evolutionary Genetics: A Primer. Menlo Park: The Benjamin/Cummings Publishing Company Inc., 1982.

25. Cvjeticanin S, Marinkovic D. Genetic variability in the group of patients with congenital hip dislocation. Genetika 2005; 41(8): 1-5.

26. Kayser M, Liu F, Janssens AC, Rivadeneira F, Lao O, van Duijn K, Vermeulen M, Arp P, Jhamai MM, van Ijcken WF, den Dunnen JT, Heath S, Zelenika D, Despriet DD, Klaver CC, Vingerling JR, de Jong PT, Hofman A, Aulchenko YS, Uitterlinden AG, Oostra BA, van Duijn CM. Three genomewide association studies and a linkage analysis identify HERC 2 as a human iris color gene. Am J Hum Genet 2008; 82(2):411-423.

27. Marinkovic D, Spremo B, Ilic M. Studies of human population-genetic variation. I. Comparisons of homozygous recessive traits in attendants of special and regular schools in SR Serbia. Arch Biol Sci 1990; 42(3-4): 8-9.

28. Marinkovic DM, Cvjeticanin S. Studies of human population-genetic variation. II. The frequencies of $\mathrm{ABO}$ blood types and homozygous re- cessive traits among top sportsmen and young intellectuals. Arch Biol Sci 1991; 43(1-2): 5-6.

29. Ristic, S, Sepcic J, Kapovic J, BrajenovicMilic B, Materljan E, Rudez J. Analysis of some phenotypic systems in a sample of multiple sclerosis patients. Balkan J Med Genet 2000; 3(3):11-18.

30. Marjanovic D, Marinkovic D, Markovic S. Population genetic study of diabetes mellitus. Acta Biol Iug Genetika 1992; 24(2):81-88.

31. Tomic V, Jankovic S, Marinkovic D. Genetic homozygosity and the occurrence of allergic bronchial asthma. Acta Biol Iug Genetika 1994; 26(3):51-56.

32. Harris H, Kalmus $H$. The measurement of taste sensitivity to phenylthiourea (PTC). Ann Eugen $1949 ; 15(1)$ : 32-45.

33. Ishihara S. Test for Color-Blindness. Tokyo: Kenehara Shuppa Company, Ltd., 1973.

34. Pesut D, Radosavljevic-Asic G, Marinkovic D. Tonsillectomy and lung cancer occurrence - negative correlation. In: Antypas G, Ed. Proceedings of the International Congress for Lung Cancer, Athens, 22-26 June, 1994. Bologna: Monduzzi Editore 1994/95;235-238. 